\title{
Yvonne Hettler. 2018. Salienz, Bewertung und Realisierung regionaler Sprachmerkmale in Bremen und Hamburg (Deutsche Dialektgeographie 124). Hildesheim: Olms. 378 S.
}

Besprochen von Carolin Kiesewalter: Philipps-Universität Marburg, Forschungszentrum Deutscher Sprachatlas, Pilgrimstein 16, D-35032 Marburg, E-Mail: Carolin.Kiesewalter@staff.uni-marburg.de

https://doi.org/10.1515/zrs-2020-2033

Mit ihrer in enger Verbindung zum Projekt „Sprachvariation in Norddeutschland“ (SiN) entstandenen Dissertationsschrift hat Yvonne Hettler einen bedeutenden Beitrag zur hörerzentrierten Regionalsprachenforschung ${ }^{1}$ geleistet. Ziel dieser Disziplin ist die Erforschung des laienlinguistischen Wissens zu modernen deutschen Regionalsprachen. Fassbar wird dieses Wissen über „Hörerurteile“ im Sinne von Veräußerungen sprachbezogener Wissensbestände (mit mittelbar oder unmittelbar vorausgegangenen Akten der Sprachwahrnehmung und -bewertung; vgl. Purschke 2011). In diesem Kontext von besonderem Interesse sind seit jeher Hörerurteile über regionalsprachliche Einzelphänomene, da deren subjektive Beurteilung nachweislich nicht nur die hörerseitige Einschätzung von Äußerungen, Sprechern und ganzen Interaktionssituationen beeinflussen kann, sondern auch die sprecherseitige Variantenwahl und somit den Sprachgebrauch. Was den Forschungsstand zum phänomenbezogenen Hörerurteil anbelangt, so ist die lang erwartete theoretische Fundierung rezent durch Purschke (2011) erfolgt. In empirischer Hinsicht sind jedoch trotz zunehmender Bemühungen ${ }^{2}$ bei Weitem noch nicht alle Desiderata behoben. Hier lässt sich nun mit Hettler (2018) ein beachtlicher Fortschritt verbuchen, denn sie legt Ergebnisse aus einer der bislang umfassendsten einschlägigen Erhebungen vor.

Den zentralen Gegenstand dieser Erhebung bilden Hörerurteile über 33 Phänomene des in Bremen bzw. Hamburg gebräuchlichen Regiolekts - darunter nicht nur phonetisch-phonologische, sondern auch (in der einschlägigen For-

1 Hettler (S. 12) selbst spricht von „Wahrnehmungsdialektologie“ und „perzeptive[r] Dialektologie“. Diese Begriffe sind derzeit gängig, greifen aber in doppelter Hinsicht zu kurz, denn die einschlägige Forschung zielt auf die Wahrnehmung und Bewertung von regional gefärbter Sprache auch jenseits des Dialektbereichs ab (vgl. Kiesewalter 2019: 27).

2 Vgl. z.B. die hörtestbasierten Untersuchungen von Herrgen \& Schmidt (1985, westmitteldeutsche Merkmale), Kiesewalter (2011, neuhessische Merkmale; 2019, nordniederdeutsche, obersächsische u. mittelbairische Merkmale), Lorenz (2014, ostwestfälische Merkmale) und Elmentaler \& Rosenberg (2015, SiN-Projekt, norddeutsche Merkmale). 
schung bislang stärker vernachlässigte) morpho-syntaktische und lexikalische Varianten. Mit diesen Urteilen sollen unterschiedlichste Aspekte der laienseitigen Phänomen-Bewertung sichtbar gemacht und aufeinander bezogen werden. Zudem soll überprüft werden, ob die Bewertungen mit außersprachlichen Faktoren in Form von sozio-demographischen Charakteristika der Beurteilenden korrelieren. Schließlich will Hettler Zusammenhänge zwischen der hörerseitigen Bewertung und dem sprecherseitigen Gebrauch der Regionalismen eruieren.

Was die Umsetzung dieses komplexen Vorhabens anbelangt, zeichnet sich Hettlers Untersuchung dadurch aus, dass sowohl über eine quantitative (hörtestbasierte) als auch über eine qualitative (introspektionsbasierte) Erhebung von Hörerurteilen operiert wird. Ein solches Vorgehen ist äußerst aufwendig, verspricht aber umso validere Ergebnisse, da es vom jeweiligen Mehrwert unterschiedlicher Datenarten profitiert, so dass letztlich ein umfassenderes Gesamtbild vom Gegenstand gezeichnet werden kann. Nach der Darlegung theoretischer Grundlagen und einem kurzen Forschungsüberblick mit Fokus auf hörtestbasierten Untersuchungen im deutschsprachigen Raum (Kap. 2 und 3) sowie der Vorstellung der untersuchten Einzelphänomene (Kap. 4) kommt Hettler in Kapitel 5 auf die Methodik ihrer Erhebungen zu sprechen. Die quantitative Erhebung besteht in einer hörtestbasierten Einzelbefragung von insgesamt 98 Bremer und Hamburger Informanten, wobei nicht nur Angehörige beider Geschlechter, sondern auch Informanten aus unterschiedlichen Alters-, Schulabschluss- und Berufsgruppen vertreten sind. Durch dieses Setting kann die Untersuchung mit einer weitaus höheren Repräsentativität der Gewährspersonen punkten als viele andere quantitative Hörtestuntersuchungen, in denen ausschließlich Schüler oder Studierende befragt wurden, und es werden auch außersprachliche Variablen überprüfbar, die in bisherigen Tests ausgeklammert blieben.

Im Hörtest beurteilen die Informanten eine Reihe von Stimuli, die in jeweils einem der 33 Untersuchungsphänomene vom Standarddeutschen abweichen. Gemäß der Instruktion der Probanden soll zunächst angegeben werden, „was an den gehörten Sätzen [...] kein ,reines Hochdeutsch““ (S. 108) ist. Daraufhin sollen subjektiv dialektale Merkmale als Antwort auf die Frage „Wo spricht man so?“ (S. 109) regional verortet werden. Im Anschluss fragt Hettler dann: „Wie finden Sie es, wenn jemand so spricht?“ und präzisierend danach, „ob das Gehörte [...] ,schlechtes“ oder ,falsches“ Deutsch sei“(S. 109). Die Frage „Haben Sie den Drang, jemanden zu korrigieren, wenn er so spricht?“ (S. 109) schließt den Test ab. Die starke Ähnlichkeit dieser Testbatterie zu der des SiN-Projekts bietet den Vorteil einer optimalen Vergleichbarkeit von Hettlers Daten mit dem noch umfassenderen projektinternen Datenkorpus. Dass pro Informant gleich mehrere unterschiedliche phänomenbezogene Urteile erhoben werden, macht außerdem eine weitaus detailliertere Rekonstruktion zugrunde liegender Konzepte möglich, als 
das in bisherigen Untersuchungen der Fall war. Als nicht ganz unproblematisch gestaltet sich allerdings die - wiederum an der Tradition des SiN-Projekts orientierte - Interpretation der Urteile. Mit Verweis auf Purschke (2011) will Hettler (S. 30) anhand der Antworten auf die erste Frage direkt die „Salienz“ im Sinne der wahrnehmungsbedingten Auffälligkeit der Phänomene bestimmen; die übrigen Urteile werden mit der „Pertinenz“ im Sinne der evaluativ-kognitiv bedingten Relevanz der Phänomene gleichgesetzt. Das ist in sich v.a. deshalb nicht schlüssig, weil Hörerurteile nach Purschke (2011: 50-87) immer auf einer Kombination von Prozessen der Wahrnehmung (> Salienzurteil) einerseits und der kognitiven Verarbeitung/Evaluation (> Pertinenzurteil) andererseits beruhen. Eine getrennte Erhebung von Salienz- und Pertinenzurteilen ist demnach (mit derzeit gängigen Hörtestverfahren) unmöglich. In der Konsequenz bleibt Hettlers Interpretation der erhobenen Urteile im Kern unscharf - das schmälert aber nicht die beeindruckende Fülle an empirisch fundierten Ergebnissen, die die Autorin in Kapitel 6, allein auf Basis ihrer quantitativen Erhebung, zum Gegenstand vorlegt.

So werden durch eine Analyse der Urteile unterschiedliche Wissensbestände zu den 33 untersuchten Phänomenen sichtbar (Kap. 6.1-6.5). Anhand der Anzahl der Nennungen eines Phänomens als hochdeutschabweichend lassen sich etwa Trends bezüglich der subjektiven Dialektalität der Merkmale aufzeigen. Merkmale wie die / $\varepsilon: /-H e b u n g$ in <Mädchen> wurden z.B. von weniger als $25 \%$ der Befragten als hochdeutschabweichend beurteilt, Merkmale wie die /g/-Spirantisierung in <Hamburg> erzielten bereits entsprechende Nennungsraten von bis zu $50 \%$, für wiederum andere Phänomene ergaben sich Raten von über $75 \%$. $^{3}$ Des Weiteren belegt Hettler (Kap. 6.2), dass Einzelphänomene als Trigger für die regionale Verortung von Sprechern fungieren können, und deckt dementsprechend die subjektive Regionalität der Phänomene auf. Hier zeigt sich u.a., dass tatsächlich großräumig in ganz Norddeutschland gebräuchliche Merkmale wie z.B. /t/-Lenisierungen und /g/-Spirantisierungen von vielen Befragten explizit mit Hamburg assoziiert werden - was mit dem medial gestützten Stereotyp von Hamburg als Zentrum des norddeutschen Kulturraums korrespondiert (S. 145). Was die affektive Bewertung der Merkmale anbelangt, so kann Hettler (Kap. 6.3) „tendenziell positiv“, „indifferent“ und „tendenziell negativ“ beurteilte Phänomene differen-

3 Hettler (Kap. 6.1) spricht in diesem Zusammenhang vom „prozentualen Salienzgrad“ der Merkmale bzw. von einer unterschiedlich „hohe[n] Auffälligkeit“ und von „hochgradig salient[en] bis gar nicht salient[en]" Merkmalen. Das ist etwas unpräzise, insofern es sich bei den zugrundeliegenden Daten nicht um graduelle Urteile handelt, wie sie in Skalentests erhebbar sind. Ermittelt wurde das Maß an interindividueller Bedeutsamkeit entsprechender Phänomene (in Hinblick auf die Frage der subjektiven Dialektalität), nicht aber der individuell empfundene Grad phänomenspezifischer Bedeutsamkeit. 
zieren. Vorrangig als „schön, sympathisch, süß ...“ (S. 150-151) bewertet werden z.B. stereotype niederdeutsche Relikte wie das alveolare /s/ vor Plosiv, da solche Merkmale vom Prestige des Plattdeutschen als vom Aussterben bedrohten Kulturguts profitieren. Eher stigmatisiert scheinen hingegen viele morpho-syntaktische Phänomene zu sein, wie z.B. der <wie>-Komparativ (<besser wie >) oder die standardabweichende Verwendung von $<$ nach $>$ (<nach Müllers $>$ ). Sie haben sich im Schulunterricht zu Fehlerstereotypen entwickelt und werden daher häufig gar nicht als regionale Spezifika interpretiert, sondern als Zeichen mangelnder Bildung (S. 145 und 150). In der Konsequenz sind es auch die „Elemente der Grammatik [, die insgesamt] eher als korrekturbedürftig [...] charakterisiert werden als lautliche Merkmale“ (S. 150). Über die systematische Quantifizierung unterschiedlicher Urteilskombinationen gelangt Hettler (Kap 6.5) schließlich zu einer Differenzierung von acht Merkmalkategorien (z.B. [s] vor Plosiv, /j/-Affrizierung u.a. = + subjektiv dialektal, + subjektiv regional [d.h. hamburgisch/bremisch], + prestigeträchtig, - korrekturbedürftig vs. <besser wie>, <nach Müllers> u.a. = + subjektiv dialektal, - subjektiv regional, - prestigeträchtig, + korrekturbedürftig). Hiermit kann die Autorin die Mehrdimensionalität von phänomenspezifischen Konzepten (vgl. Eckerts 2008 sogenannte „indexical fields“) aufzeigen und wesentliche Konzeptbereiche sichtbar machen - wenngleich sie selbst diesen Schluss nicht zieht. ${ }^{4}$

Den zweiten Teil ihrer quantitativen Untersuchung widmet Hettler (Kap. 6.6) der Frage nach etwaigen Zusammenhängen zwischen außersprachlichen Faktoren und phänomenbezogenen Hörerurteilen. Solche Zusammenhänge sind aus theoretischer Sicht unbedingt erwartbar, aber empirisch bislang nur ansatzweise erforscht. Hettler leistet hier also wiederum Pionierarbeit, wenn sie mit statistischen Signifikanzen belegt, dass die subjektive Dialektalität zahlreicher Regionalismen mit dem Alter, Bildungsniveau respektive Beruf der Beurteilenden korreliert. ${ }^{5}$ In der Mehrheit der Fälle entspricht die Effektrichtung den Erwartungen. In einer abschließenden Differenzierung zweier „Hörertypen“ (S. 185-187) werden entsprechende Trends noch einmal verdeutlicht: Hörer, die überdurchschnittlich viele Phänomene als hochdeutschabweichend verbalisierten, sind mehrheitlich (ehemalige) Studierende jüngeren bis mittleren Alters sowie vielfach in sprach-

\footnotetext{
4 Vgl. stattdessen Hettler (S. 170): „Die ermittelten Kategorien zeigen die Bandbreite an Bewertungskombinationen auf, denen sprachliche Merkmale in Bezug auf ihre Salienz unterliegen, und stellen somit unterschiedliche Ausprägungen der Pertinenz dar.“

5 Im Kontext der Gendervariable lassen sich keine signifikanten Bewertungsunterschiede nachweisen und auch herkunftsspezifische Differenzen treten zumindest bei der Beurteilung der Dialektalität nur vereinzelt auf. Letzteres wird von Hettler plausibel erklärt, indem sie auf die großräumige Verbreitung der meisten Untersuchungsphänomene verweist (s. Kap. 6.1 und 6.6.2).
} 
zentrierten Berufen tätig. Hörer, die besonders wenige Normabweichungen angaben, sind i.d.R. über 65 Jahre alt, haben maximal einen Realschulabschluss und sind bzw. waren eher mit manuellen oder zumindest nicht sprachzentrierten Tätigkeiten betraut.

In Kapitel 7 präsentiert die Autorin die Ergebnisse ihrer qualitativen Erhebung. Diese Erhebung stützt sich auf jeweils zehn weitere Informanten aus Hamburg und Bremen, die ebenfalls den Hörtest absolvierten, zuvor jedoch ausführlich zum Sprachgebrauch in Bremen und Hamburg interviewt wurden, wobei auch offen nach typischen Sprachmerkmalen der Stadtsprachen gefragt wurde. Auf Basis dieser introspektionsbasierten Daten leistet Hettler (Kap. 7.3) zunächst eine systematische Rekonstruktion der Konzepte zum Bremischen und Hamburgischen. Dabei werden Wissensbestände zur Bezeichnung sowie zur diatopischen, diastratischen und diaphasischen Einordnung entsprechender Varietäten ebenso aufgedeckt wie konzeptspezifische Werteurteile und Sprachmerkmale.

Da von allen Informanten der qualitativen Untersuchung außerdem Sprachgebrauchsproben in unterschiedlich formellen Erhebungssituationen (im Einzelnen: Interview, Freundesgespräch und Vorleseprobe) gesammelt wurden, kann Hettler (Kap. 7.4) dann einen radikalen Perspektivwechsel vollziehen, der die Arbeit für die gegenwärtige Forschung wiederum besonders wertvoll macht. Für 24 lautliche und drei morpho-syntaktische Untersuchungsphänomene werden intersituative Gebrauchsfrequenzen präsentiert, die in umfangreichen Variablenanalysen ermittelt wurden. ${ }^{6}$ Die objektsprachlichen Ergebnisse werden zunächst sorgfältig isoliert und phänomenweise besprochen, wobei v.a. die intersituative Bandbreite der Frequenzen sowie Korrelationen mit dem situativen Formalitätsgrad, bestimmten Lexemkontexten, dem Alter und der Herkunft der Sprecher herausgearbeitet werden. Darauf aufbauend werden dann die „Bedingungen des Variantengebrauchs“ (Kap. 7.4.5) zusammengefasst.

Abschließend nimmt Hettler (Kap. 7.5) eine Synthese ihrer subjekt- und objektsprachlichen Ergebnisse vor und leitet daraus vier „Sprecher- und Hörertypen“ ab. In diesem Kontext werden z.B. die „Sprachbewussten“ und die „Regionsverwachsenen“ unterschieden. ${ }^{7}$ Erstere beurteilen im Hörtest überdurchschnittlich viele Varianten als normabweichend, hegen insgesamt ein „großes Interesse an [regional gefärbter] Sprache“ (S. 282), sind häufig jüngeren Alters und vielfach (auch berufsbedingt) fähig, subjektiv dialektale Regionalismen intersituativ zu variieren. „Regionsverwachsene“ Informanten zeichnen sich hin-

6 Sprecherweise analysiert wurden je 2500 Wörter aus den freien Gesprächen und 343 Wörter aus der Leseprobe.

7 Die übrigen zwei Typen sind die „Sprachvarianztoleranten“ und die „Durchschnittshörer“. 
gegen dadurch aus, dass sie vergleichsweise wenige Phänomene als normabweichend beurteilen, mehrheitlich älter sind und einen standardferneren Sprachgebrauch pflegen. Durch diese Kategorisierung können grundlegende Zusammenhänge zwischen Sprachbewertung, Sprachwissen und Sprachgebrauch aufgedeckt werden; teilweise wird dies aber auch durch die Komplexität des Untersuchungssettings erschwert. Hier stellt sich die Frage, ob man nicht stellenweise noch klarere Ergebnisse hätte erzielen können, wenn man auf einige Untersuchungsvariablen verzichtet hätte. Insgesamt ist es aber gerade die bislang beispiellose Verknüpfung unterschiedlichster Variablen, Methoden, Daten und Analyseverfahren, durch die Hettlers Dissertationsschrift besticht. Letztendlich gelingt hierdurch eine ganzheitliche Betrachtungsweise, die die Lektüre der Arbeit unbedingt empfehlenswert macht - und das keineswegs nur für Forschende im Bereich des phänomenbezogenen Hörerurteils, für die es sich beim vorliegenden Werk ohnehin um Pflichtlektüre handelt.

\section{Literatur}

Eckert, Penelope. 2008. Variation and the indexical field. In: Journal of Sociolinguistics 12, 453-476.

Elmentaler, Michael \& Peter Rosenberg. 2015. Norddeutscher Sprachatlas (NOSA). Bd. 1: Regiolektale Sprachlagen (Deutsche Dialektgeographie 113.1). Hildesheim: Olms.

Herrgen, Joachim \& Jürgen Erich Schmidt. 1985. Systemkontrast und Hörerurteil. Zwei Dialektalitätsbegriffe und die ihnen entsprechenden Meßverfahren. In: Zeitschrift für Dialektologie und Linguistik 52(1), 20-42.

Kiesewalter, Carolin. 2011. Zur Salienz remanenter Merkmale des Neuhessischen. In: Germanistische Linguistik 216-217, 335-369.

Kiesewalter, Carolin. 2019. Zur subjektiven Dialektalität regiolektaler Aussprachemerkmale des Deutschen (Zeitschrift für Dialektologie und Linguistik. Beihefte 179). Stuttgart: Steiner.

Lorenz, Cornelia. 2014. Zugezogene im Fokus. Sprachkontakterscheinungen im Regiolekt (Tübinger Beiträge zur Linguistik 550). Tübingen: Narr.

Purschke, Christoph. 2011. Regionalsprache und Hörerurteil. Grundzüge einer perzeptiven Variationslinguistik (Zeitschrift für Dialektologie und Linguistik. Beihefte 149). Stuttgart: Steiner. 